\title{
Behcet's disease and the neuropathic bladder: urodynamic features: case report and a literature review
}

\author{
D Porru, ${ }^{1}$ AC Pau, ${ }^{1}$ RM Scarpa, ${ }^{1}$ L Zanolla, ${ }^{2}$ A Cao ${ }^{2}$ and E Usai ${ }^{1}$ \\ ${ }^{1}$ Clinica Urologica, Ospedale SS. Trinità, Cagliari; ${ }^{2}$ Clinica Pediatrica $2 a$, Ospedale Microcitemico, Via Jenner, \\ Cagliari; Italy
}

\begin{abstract}
We report on a 16-year old patient with a neuropathic bladder secondary to Behcet's disease, which is an uncommon vasculitis usually involving venules. The genitourinary manifestations of this disease are discussed, a neuropathic bladder being a rare complication of the involvement of the nervous system. Urodynamic assessment is important when voiding dysfunction is present; three patients previously reported revealed a bladder function changing from normal detrusor to overactivity. Our patient showed early and severe involvement of the nervous system, and detrusor areflexia two years after the onset of the disease. Spontaneous voiding was restored two months after urological management (intermittent catheterization) was started.
\end{abstract}

Keywords: Behcet's disease; neuropathic bladder; urodynamic assessment

\section{Introduction}

The first presentation of Behcet's disease was made by Adamantiades at the Athenean International Congress of Opthalmology. ${ }^{1}$ A syndrome of aphthous ulcers of the mouth and genitals was described, associated with skin lesions and hypopyon uveitis. Behcet's disease is a vasculitis with a prevalent involvement of venules. Oral and genital recurrent ulcers are commonly seen, as well as ocular lesions and other dermatological lesions. It is more common in males than in females (ratio 5:1), and has a prevalent incidence in Japan and in Mediterranean countries. ${ }^{2}$ The pathogenesis is not clear, but the production of immunocomplexes is involved. Ocular lesions are the most prominent component of Behcet's triad, because of their usually severe relapsing clinical course and poor prognosis As regards the CNS, meningoencephalitis and intracranial hypertension can be present, and the symptoms are usually similar to multiple sclerosis or to a pyramidal syndrome; ${ }^{3}$ the condition of some patients may suddenly become extremely poor and neurological complications may appear only a few weeks before death.

\section{Case report}

A 16-year-old young man, with Behcet's disease since the age of 14 , complained of micturition disorders 6 months before our observation: hesitancy, intermittency, incomplete voiding, and eventually urinary retention. Physical examination revealed severe ocular

Correspondence: D Porru, Via Lunigiana 1, 09122 Cagliari, Italy lesions (uveitis), spastic paraparesis, normal anal sphincter tone; the bulbocavernosus reflex was present, the deep tendon reflexes were normal. Scrotal ulcers and labial aptha were present. MRI revealed mild atrophy of all segments of the spinal cord and of the cauda equina (Figure 1); also encephalic lesions

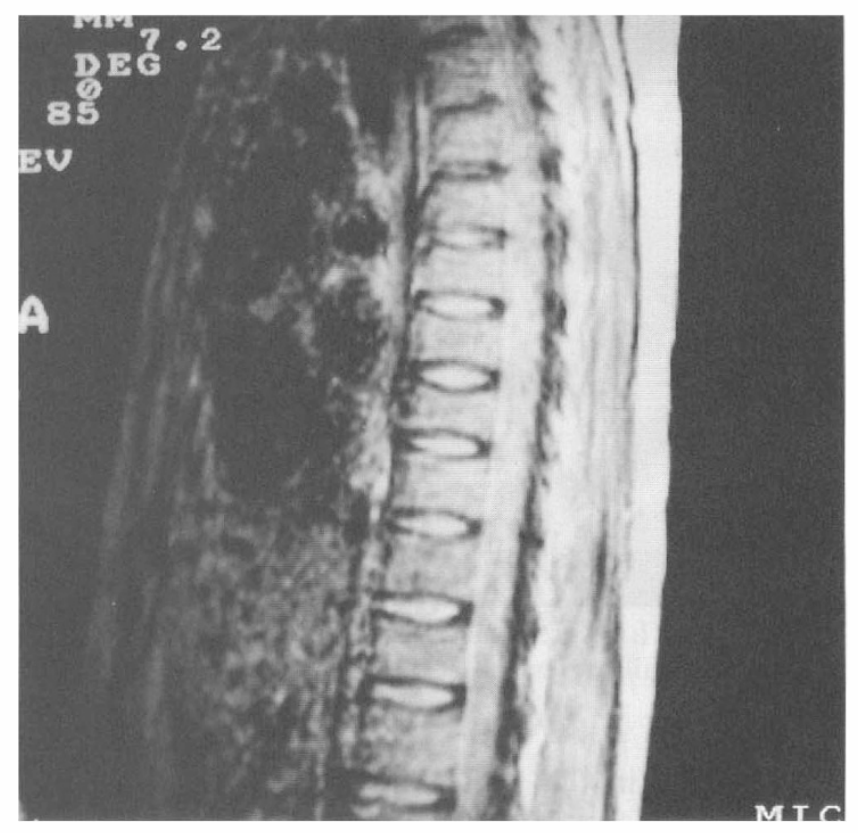

Figure 1 MRI of spinal cord: mild atrophy of cervical, dorsal, lumbosacral spinal cord and cauda equina 


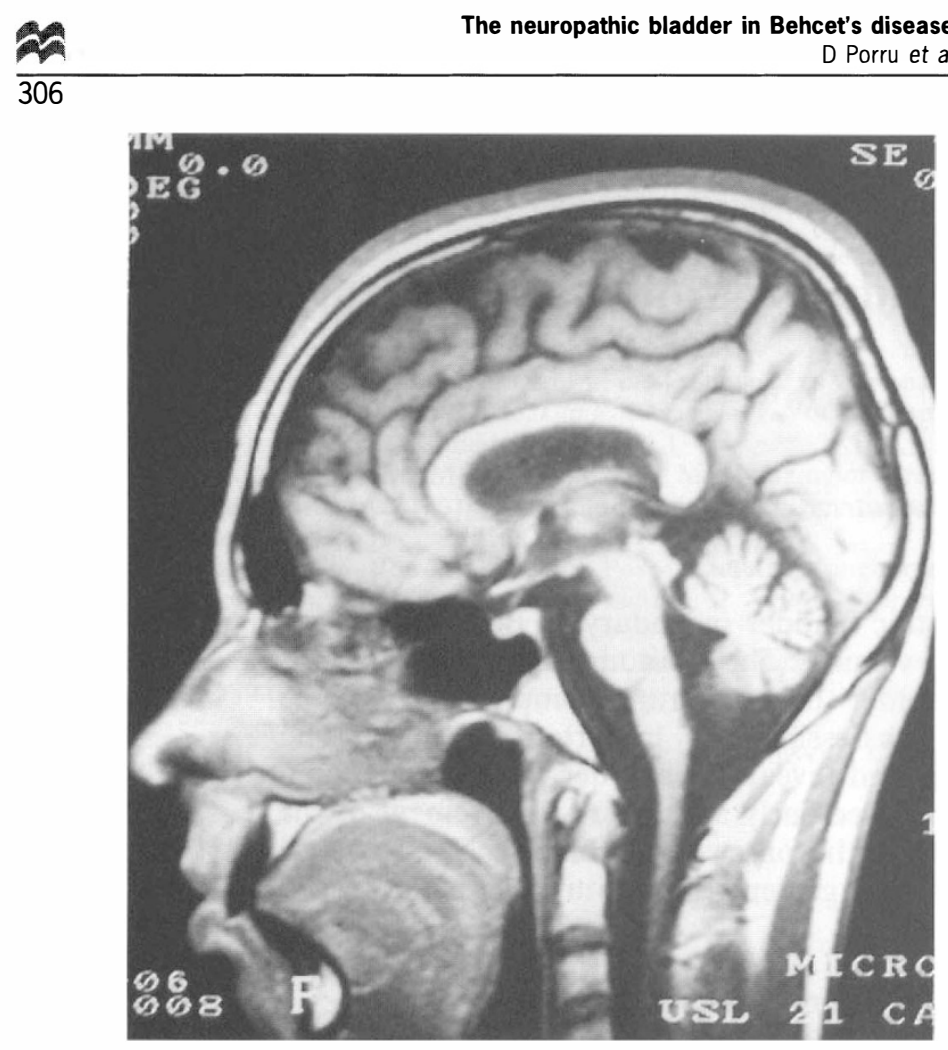

Figure 2 MRI: cortical atrophy, with secondary enlargement of both ventricles

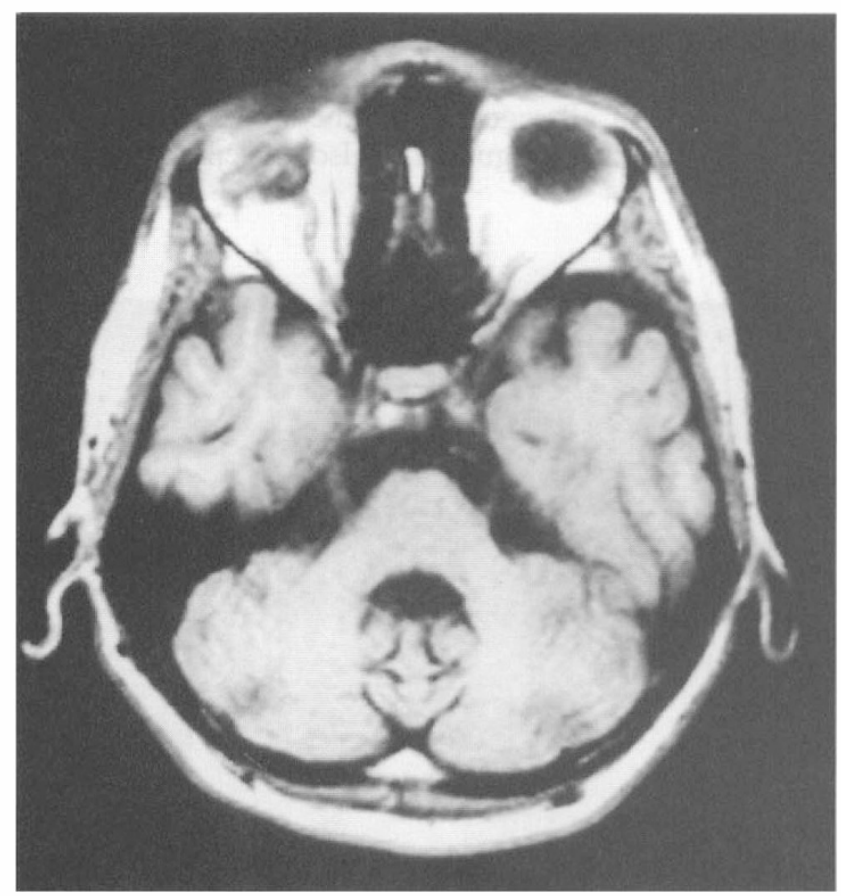

Figure 3 MRI: severe structural and morphologic lesions of the right eyeball are evident

were located in the white matter of the right frontal lobe, as well as in the diencephalon, bulb and pons, probably as a result of a previous vasculitis; cortical atrophy caused enlargement of both ventricles (Figure
$2)$; the right eyeball showed evident structural and morphological lesions (Figure 3).

Ultrasound showed a normal upper urinary tract. Urodynamic investigation during the storage phase revealed detrusor areflexia and reduced bladder sensation; the bladder capacity was $400 \mathrm{ml}$, with a normal compliance and a normal EMG pattern; the bethanecol supersensitivity test was positive. Clean self intermittent catheterisation was started six times a day, and subsequently changed as frequently as was necessary to ensure that the residual urine was less than $400 \mathrm{ml}$, thus preventing bladder overdistension. At 3 months the patient started to void spontaneously, intermittent catheterisation was performed once a day, with a mean residual urine of $150 \mathrm{ml}$; bacteriuria was absent. Six months after first observation the spastic paraparesis was stable, urodynamics revealed detrusor instability, a normal EMG-pattern, and impaired detrusor contractility, with incomplete bladder emptying. The patient was continent with oxybutinin $5 \mathrm{mg}$. twice daily and intermittent catheterisation twice daily, performed after spontaneous voiding. The subsequent behaviour of the voiding system seemed to follow the same changeable course of the general disease. The patient was positive for B51 group of HLA antigens. The patient showed an improvement in the spastic paraparesis within 3 months after the first observation, and at the same time regained detrusor contractility after starting clean intermittent catheterization. This improvement was maintained until his last visit, in October 1995.

\section{Discussion}

The important diagnostic symptoms of Behcet's multisystem disease are: oral and genital (scrotal) ulceration, and ocular lesions. As regards the urinary tract, in addition to genital ulceration, urethritis and, more frequently, epididymitis may occur, sometimes recurrently. Occasionally, there is an association between relapses of the uveitis and of the oral and genital manifestations. A patient suffering from urethro-vaginal fistula and Bechet's syndrome was described. ${ }^{2}$ Some authors, ${ }^{4}$ in a prospective study of 100 consecutive patients with newly diagnosed Behcet's disease, observed that $89 \%$ of the males had experienced scrotal ulceration, $6 \%$ had epidydimitis, and $3 \%$ sterile urethritis. Behcet's disease located in the bladder ${ }^{5}$ was found to be a rare cause of hematuria. To our knowledge, only three cases of neuropathic bladder due to neuro-Behcet disease are reported in the international literature so far. ${ }^{6}$ Two of the patients complained of urinary frequency and incontinence; a urodynamic study revealed overactive detrusor activity in the storage phase and normal or impaired detrusor activity during voiding. The third patient complained of urinary retention, with an areflexic detrusor in the voiding phase. All of the patients showed detrusorsphincter dyssynergia. Repeated urodynamic studies revealed that bladder function changed from normal 
detrusor to overactivity in the storage phase. In our case, repeated urodynamic monitoring revealed the same changing behaviour from 'areflexia' to overactivity with a normal EMG-pattern: this suggests that the initial findings could have been due to acontractility, because of a temporary detrusor decompensation. However, neurological involvement in this case was progressive and involved all segments of spinal cord, including the conus medullaris, therefore an abnormal nervous control of micturition is likely. The urodynamic changes observed after 3 months could be due to the effect of immunosuppressive therapy with cortisone and methotrexate on the neurological lesions.

Involvement of the nervous system is particularly significant during the course of Behcet's syndrome for its prognostic value, since it is a clear indication of worsening of the disease. ${ }^{7,8}$ indicated that neurological involvement was present in approximately one-quarter of all cases in the literature. Several reports in the literature have commented on the underlying similarity to multiple sclerosis; ${ }^{9}$ among the 50 cases in the literature reviewed by Gherardi, ${ }^{10}$ there were eight examples of brain stem syndromes and 12 of the multiple sclerosis-like form. In practice there are many differences between cases apparently of the same type, and in the same patient between a first and a second episode of neurological complications. The general condition of the patients is frequently poor, and the later course of the illness in fatal cases changes irregularly, at times even dramatically so.

The most significant neuropathological changes in neuro-Behcet's disease are small foci of complete or incomplete necrosis disseminated throughout the brain and spinal cord, with a predilection for the brain stem (especially the midbrain), hypothalamus, internal capsule and basal ganglia; ${ }^{11}$ the white matter is preferentially affected. This is the true mark of the disease. The localization of these lesions should prompt the clinician to search for early voiding symptoms, and to carefully investigate the bladder function of the affected patient.

\section{Conclusions}

In our experience a neuropathic bladder due to neuroBehcet disease changed its urodynamic features, from detrusor areflexia to detrusor instability, and impaired contractility, and subsequently changed following the course of the disease, depending on the level of the spinal cord and cerebral lesions, as is so often observed in multiple sclerosis patients. Periodic clinical and urodynamic follow-up is therefore important in the urological management of this disease.

\section{References}

1 Adamantiades B. Sur un cas d'iritis à hypopyon rècidivante. Ann Oculist 1931; 168: 271 - 275.

2 Waidelich RM, Brunschweiger SM, Schmeller NY. Urethrovaginal fistula in Behcet disease. Urologe 1994; 33(2): 163-166.

3 Stanescu A, Petrescu A, Kreindler A. The meningo-encephalomyelo-polyradicular form of Behcet's disease. An anatomicoclinical case. Rom J Neurol Psychiatry 1990; 28(4): 315-320.

4 Kirkali Z, Ygitbasi O, Sasmaz R. Urological aspects of Behcet's disease. Br. J. Urol. 1991; 67(6): 638-639.

5 Carnovale A, Nuzzi L, Uomo G. A rare cause of hematuria. Behcet disease located in the bladder. Minerva Urologica $e$ Nefrologica 1985; 37(2): 195-197.

6 Nakagawa $\mathrm{H}$ et al. Three cases of neurogenic bladder due to neuro-Behcet disease. Nippon Ninyokika Gakkai Zasshi 1994; 85(9): $1399-1402$.

7 Hermann C, Jr. Involvement of the nervous system in relapsing uveitis with recurrent genital and oral ulcers. Arch Neurol Psychiat (Chic) 1953; 69: 399-403.

8 Wolf SM, Schotland DL, Phillips LL. Involvement of the nervous system in Behcet's syndrome. Arch Neurol (Chic) 1965; 12: $315-$ 325 .

9 Asayama R, Shimizu A, Akutagawa T. A case of Behcet's disease with symptoms of multiple sclerosis. J Clinical Ophtal 1963; 17: $15-21$.

10 Gherardi D, Nazzaro P. Le complicanze neurologiche del morbo di Behcet, Lav Neuropsichiat 1966; 37: $333-408$.

11 Alemà $G$, Bignami $A$. Involvement of the nervous system in the Behcet's disease. International Symposium on Behcet's disease. Karger, Basel-New-York 1966; pp 52-66. 